\title{
POSTER
}

\section{Intérêts de l'imagerie CBCT dans la gestion des complications en chirurgie orale}

\section{Moreau $\mathbf{N}^{1,2}$, Ejeil AL', Salmon B ${ }^{1,3}$}

1 - Service d'odontologie, Hôpital Bretonneau, AP-HP, Hôpitaux Universitaires Paris Nord Val de Seine, Paris, France

2 - INSERM UMR 894, Centre de Psychiatrie et Neurosciences, Université Paris Descartes Sorbonne Paris Cité, Hôpital Sainte-Anne, Paris

3 - EA 2496, Université Paris Descartes Sorbonne Paris Cité, Faculté de Chirurgie Dentaire, Montrouge, France

\section{Introduction}

Facile d'accès, rapide et assurant des images de qualité au prix d'une dose raisonnable, l'exploration 3D par Cone Beam Computed Tomography [CBCT] optimise la pratique courante de la chirurgie orale et maxillo-faciale (Mupparapu \& Nadeau 2016).

Outre l'apport indéniable et aujourd'hui bien documenté de cette technologie dans le bilan préopératoire en chirurgie orale (exploration fine des relations anatomiques d'une dent avec les structures vasculo-nerveuses avoisinantes, planification pré-implantaire au sein d'un faible volume osseux,...), il convient de souligner également l'intérêt non négligeable de l'imagerie 3D dans la gestion per- et post opératoire des complications des actes de chirurgie orale.

\section{Observation}

Ce travail propose d'illustrer à travers plusieurs cas cliniques, la contribution de l'imagerie CBCT dans la gestion de différentes complications chirurgicales. Dans chacun des cas présentés, l'indication judicieuse de cet examen complémentaire a permis soit de faciliter la localisation per ou post-opératoire d'une structure d'intérêt (table osseuse, apex dentaire, fragment d'instrument....) soit d'apporter un éclairage diagnostique à des douleurs post-opératoires atypiques et inexpliquées.

\section{Discussion}

À l'instar des autres examens radiographiques, l'imagerie CBCT se doit de répondre à des principes universels de précaution et de bonne pratique. Ainsi le position paper de l'académie américaine de radiologie orale et maxillo-faciale (American Academy of Oral and Maxillofacial Radiology), comme les recommandations européennes, rappellent les responsabilités du praticien qui prescrit et pratique un examen $\mathrm{CBCT}$ et enjoint à respecter les principes fondamentaux de radioprotection (justification et «ALARA » [As Low As Reasonably Acheivable] ) (Carter et al. 2008, Guidelines SEDENTEXCT 2012).

Dans le cadre spécifique de la gestion per- et post-opératoire des complications en chirurgie orale, l'imagerie tridimensionnelle extemporanée est susceptible d'éclairer une situation complexe en améliorant la qualité et la rapidité de la prise en en charge tout en facilitant l'orientation in situ au cours de l'acte chirurgical.

This is an Open Access article distributed under the terms of the Creative Commons Attribution License 4.0, which permits unrestricted use, distribution, and reproduction in any medium, provided the original work is properly cited. 


\section{Conclusion}

Si l'analyse rigoureuse du bilan préopératoire permet de prévenir et d'anticiper les difficultés chirurgicales, l'imagerie 3D présente un intérêt indéniable dans la gestion des complications.

\section{Références}

Carter L, Farman AG, Geist J, et al. American Academy of Oral and Maxillofacial Radiology executive opinion statement on performing and interpreting diagnostic cone beam computed tomography. Oral Surg Oral Med Oral Pathol Oral Radiol Endod 2008;106:561.

Guidelines SEDENTEXCT 2012. http://www.sedentexct.eu/content/guidelines-cbct-dental-and-maxillofacial-radiology. Mupparapu M, Nadeau C. Oral and maxillofacialimaging. Dent Clin North Am. 2016;60(1):1-37. 